\title{
An Econometric Study Of Vine Copulas
}

\author{
D. Guégan*and P.A. Maugis ${ }^{\dagger}$ \\ PSE, Université Paris 1 Panthéon-Sorbonne, \\ 106 boulevard de l'Hopital 75647 Paris Cedex 13, France
}

\begin{abstract}
We present a new recursive algorithm to construct vine copulas based on an underlying tree structure. This new structure is interesting to compute multivariate distributions for dependent random variables. We proove the asymptotic normality of the vine copula parameter estimator and show that all vine copula parameter estimators have comparable variance. Both results are crucial to motivate any econometrical work based on vine copulas. We provide an application of vine copulas to estimate the $V a R$ of a portfolio, and show they offer significant improvement as compared to a benchmark estimator based on a $G A R C H$ model.
\end{abstract}

Keywords: Vines Copulas - Conditional Copulas - Risk management. JEL: D81 - C10 - C40 - C 52

\section{Introduction}

For almost ten years now, copulas have been used in econometrics and finance. They became an essential tool for pricing complex products, managing portfolios and evaluating risks in banks and insurance companies. For instance, they can be used to compute $\operatorname{VaR}$ (Value at Risk) and ES (Expected shortfall), Artzner et al. (1997). Moreover, copulas appear to be a very flexible tool, allowing for semi-parametric estimation, fast parameter optimisation and time varying parameters. These advantages make them a very interesting tool, although one major shortcoming is their use in high dimension. Indeed, elliptical copulas can be expended to higher dimension, but they are unable to model for financial tail dependences (Patton, 2009), and the Archimedean copulas are not satisfactory

*email: dominique.guegan@univ-paris1.fr.

$\dagger$ e-mail: pierre-andre.maugis@malix.univ-paris1.fr. 
as models to describe multivariate dependence in dimensions higher than 2 (Joe, 1997).

The objective of this paper is twofold. In a first step we introduce a new recursive algorithm to construct vine copulas. This new type of copulas permits to estimate the dependence between random variables in any dimensions (Joe (1997) and Bedford and Cooke (2002, 2001)). Vine copulas have already been studied by several authors, focussing on information optimisation and algorithm efficiency. They are introduced as decomposition of a multivariate random vector density based on a graph structure called "vines", Bedford and Cooke (2002, 2001) and Cooke (1997). We propose an other approach considering an algorithm based on step-by-step factorisation of the density function in a product of bivariate copulas. This method permits to exhibit the underlying tree structure of vine copulas that will be central in the proofs of the theorems of convergence.

In a second part, we provide new result on the estimator of the vine copulas built in the previous step. Indeed, denoting $\theta$ the parameter of a vine copula, we prove the asymptotic normality of the estimator with a convergence rate of $\sqrt{T}$, where $T$ is the sample size. This new result justifies the use of vine copulas in economic applications (Aas et al. (2009), Berg and Aas (2010), Czado et al. (2009), Fischer et al. (2007), Chollete et al. (2008) and Guégan and Maugis (2010)), and also provides confidence intervals. Finally, we study the variance behavior of the estimates across vine copulas and show that any two vine copulas estimates have comparable asymptotic variance. Our result proves that all vine copula should be used, and that there are no statistical ground for favoring a subset of them over another one since they are all efficient estimators in terms of rate and speed of convergence. It confirms the fact that using all possible vine copulas permits to describe more varied dependence (Guégan and Maugis, 2010). Our results are prooved under a regular set of hypothesis commonly found when using copulas (Patton, 2009) and holds for any type of bivariate copulas, this includes conditional copulas, Markov switching copulas and mixture copulas.

The paper is organised as follow: In section 2 we construct the set of vines decomposition we work on and present its underlying tree structure. In section 3 we derive the asymptotic properties of the vines as estimators and bound their relative variance. Section 4 presents an application and section 5 concludes.

We now recall the definition of a copula, Sklar (1959). Let $X=\left(X_{1}, X_{2}, \ldots, X_{n}\right)$ be a vector of random variables, with joint distribution $F$ and marginal distributions $F_{1}, \ldots, F_{n}$, then there exists a function $C$-called copula - mapping the individual distribution to the joint distribution:

$$
F\left(x_{1}, \ldots, x_{n}\right)=C\left(F_{1}\left(x_{1}\right), F_{2}\left(x_{2}\right), \ldots, F_{n}\left(x_{n}\right)\right)
$$

Let $Y$ be another vector of random variables. We call $\tilde{F}$ the distribution function of $(X \mid Y)$. Patton (2006) defines the conditional copula of $(X \mid Y)$ as the function mapping the individual distributions to the conditional distribution:

$$
\tilde{F}\left(x_{1}, \ldots, x_{n} \mid y_{1}, \ldots, y_{p}\right)=C\left(F_{1}\left(x_{1}\right), F_{2}\left(x_{2}\right), \ldots, F_{n}\left(x_{n}\right) \mid y_{1}, \ldots, y_{p}\right)
$$




\section{Vine Construction}

In this section, we introduce a new algorithm to build vine copulas. Our approach has the advantage of being able to coherently describe a large set of vine

copulas $-N=\frac{n !}{2} \prod_{i=1}^{n-3} i$ ! in dimension $n$ - while also being a simple recursive algorithm. Moreover the tree structure and the algorithm are fully recursive so they can be easily expanded to any dimension.

\subsection{Formula}

Let us consider a vector $\boldsymbol{X}=\left(X_{1}, X_{2}, \ldots, X_{n}\right)$ of random variables characterised by a joint distribution function $F_{\boldsymbol{X}}$ and we assume it has a density function $f_{\boldsymbol{X}}$. We introduce some notations:

- $\boldsymbol{X}_{-\alpha}=\left(X_{1}, \ldots, X_{\alpha-1}, X_{\alpha+1}, \ldots, X_{n}\right)$ is the set of variables except the $\alpha$-th.

- We denote $f_{\alpha}$ the density of $X_{\alpha}$. In the same fashion $f_{\alpha \mid \beta}$ is the density of $\left(X_{\alpha} \mid X_{\beta}\right), f_{-\alpha}$ is the density of $\boldsymbol{X}_{-\alpha}$ and $f_{\alpha \mid-\beta}$ is the density of $X_{\alpha} \mid \boldsymbol{X}_{-\beta}$. We use the similar notation for the distribution function $F$ : for instance $F_{\alpha \mid-\beta}$ is the distribution function of $X_{\alpha} \mid \boldsymbol{X}_{-\beta}$.

- $c_{\alpha, \beta \mid \gamma}=c_{X_{\alpha}, X_{\beta} \mid X_{\gamma}}\left(F_{X_{\alpha} \mid X_{\gamma}}\left(X_{\alpha} \mid X_{\gamma}\right), F_{X_{\beta} \mid X_{\gamma}}\left(X_{\beta} \mid X_{\gamma}\right)\right)$ is the copula density of $\left(X_{\alpha}, X_{\beta} \mid X_{\gamma}\right)$ as defined in Sklar (1959). Similarly we denote as $c_{\alpha, \beta \mid-\gamma}$ the copula density of $\left(X_{\alpha}, X_{\beta} \mid X_{-\gamma}\right)$ :

$$
c_{\alpha, \beta \mid-\gamma}=c_{X_{\alpha}, X_{\beta} \mid \boldsymbol{X}_{-\gamma}}\left(F_{X_{\alpha} \mid \boldsymbol{X}_{-\gamma}}\left(X_{\alpha} \mid \boldsymbol{X}_{-\gamma}\right), F_{X_{\beta} \mid \boldsymbol{X}_{-\gamma}}\left(X_{\beta} \mid \boldsymbol{X}_{-\gamma}\right)\right) .
$$

We also use $C$ with the same notations.

Our objective is to compute $c_{1, \ldots, n}$, the copula density associated with the vector $\boldsymbol{X}$. This will be done by factorizing $f_{\boldsymbol{X}}$ in the following form:

$$
f_{\boldsymbol{X}}=\prod_{i=1, \ldots n} f_{i} \cdot c_{1, \ldots, n}
$$

By construction for $n=2$ we have: $f_{\alpha, \beta}=f_{\alpha} \cdot f_{\beta} \cdot c_{\alpha, \beta}$. Using this property we consider the following factorisation of the joint density $f_{\boldsymbol{X}}$ : $\forall \alpha, \beta \in\{1, \ldots, n\}^{2}, \alpha \neq \beta$

$$
\begin{aligned}
f_{\boldsymbol{X}}=f_{-\alpha} \cdot f_{\alpha \mid-\alpha}=f_{-\alpha} \cdot \frac{f_{\alpha, \beta \mid-(\alpha, \beta)}}{f_{\beta \mid-(\alpha, \beta)}} & =f_{-\alpha} \cdot \frac{f_{\alpha \mid-(\alpha, \beta)} \cdot f_{\beta \mid-(\alpha, \beta)}}{f_{\beta \mid-(\alpha, \beta)}} \cdot c_{\alpha, \beta \mid-(\alpha, \beta)} \\
& =\frac{f_{-\alpha} \cdot f_{-\beta}}{f_{-(\alpha, \beta)}} \cdot c_{\alpha, \beta \mid-(\alpha, \beta)} .
\end{aligned}
$$


Formula (1) allows the computation of an $n$-variate density with a bivariate copula, two $(n-1)$-and one $(n-2)$-variate densities. Using this factorisation recursively, insuring that the denominators cancel at each step, we produce a factorisation of the $n$-variate density as a product of univariate and bivariate copula densities. Using this algorithm we can produce all $N$ possible vine copulas (Napoles, 2007) $)^{1,2}$.

At each step of the algorithm we associate a tree construction. The root of this tree is the new copula density term: $c_{\alpha, \beta \mid-(\alpha, \beta)}$ in expression (1), and the leaves are the new $(n-1)$-variates densities: $f_{-\alpha}$ and $f_{-\beta}$ in (1). The tree associated with expression (1) is:

$$
\overbrace{f_{-\alpha}}^{c_{\alpha, \beta \mid-(\alpha, \beta)}} f_{-\beta}
$$

This tree structure is also fully recursive. To each term $f_{-\alpha}$ and $f_{-\beta}$ we could apply (1), and produce trees. These trees would then be inserted inside the previous tree replacing the two leaves $f_{-\alpha}$ and $f_{-\beta}$ by the two new tree roots. We explain this mechanism further in an example.

\subsection{Example}

In this example, we illustrate the unwinding of the previous algorithm for $n=4$, providing the joint density function $f_{1,2,3,4}$. Our aim is to compute $c_{1,2,3,4}$ : the joint copula density. We describe the three steps of the algorithm using the previous notations. With this example, we detail the construction of the tree associated with a specific vine:

- First step:

$$
\begin{gathered}
f_{1,2,3,4}=\frac{f_{1,2,3} \cdot f_{1,2,4}}{f_{1,2}} \cdot c_{3,4 \mid 1,2} \\
\overbrace{f_{1,2,3}}^{c_{3,4 \mid 1,2}}
\end{gathered}
$$

\footnotetext{
${ }^{1}$ This is the number of "vine" type graph with $n$ nodes, which is also the number of vine copulas (see Bedford and Cooke $(2002,2001))$. The proof of the formula relies heavily on the graph structure of vines.

${ }^{2}$ Our algorithm can produce more varied decompositions, however we do not consider those additional copulas as they are not efficient estimators, Bedford and Cooke (2002, 2001).
} 
- Second step: we apply the relationship (1) to $f_{1,2,3}$ and $f_{1,2,4}$ and produce two sub-trees:

$$
\begin{gathered}
f_{1,2,3}=\frac{f_{1,2} \cdot f_{1,3}}{f_{1}} \cdot c_{2,3 \mid 1} \\
\overbrace{f_{1,2}}^{c_{2,3 \mid 1}}
\end{gathered}
$$

and

$$
\begin{gathered}
f_{1,2,4}=\frac{f_{1,2} \cdot f_{2,4}}{f_{2}} \cdot c_{1,4 \mid 2} \\
\overbrace{f_{2,4}}^{c_{1,4 \mid 2}}
\end{gathered}
$$

- Third step: We merge formulas (2),(3) and (4), we simplify the $f_{1,2}$ term and we expand the bivariate densities using the formula: $f_{\alpha, \beta}=f_{\alpha} \cdot f_{\beta} \cdot c_{\alpha, \beta}$. We also merge the trees and underline the simplified term in the tree.

$$
f_{1,2,3,4}=f_{1} \cdot f_{2} \cdot f_{3} \cdot f_{4} \cdot c_{1,3} \cdot c_{1,2} \cdot c_{2,4} \cdot c_{2,3 \mid 1} \cdot c_{1,4 \mid 2} \cdot c_{3,4 \mid 1,2}
$$

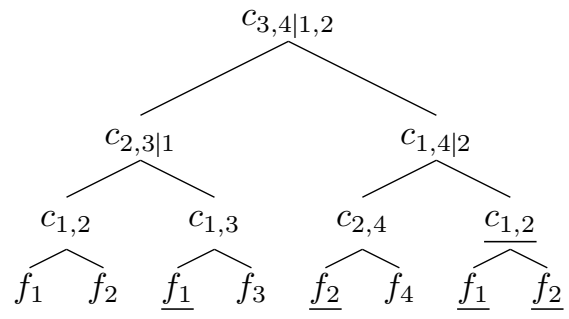

We have now factorised the density $f_{1,2,3,4}$ into a product of four univariate densities and six bivariate copula densities. By construction, this means that the copula density of $\left(X_{1}, X_{2}, X_{3}, X_{4}\right)$ can be factorised as follows:

$$
c_{1,2,3,4}=c_{1,3} \cdot c_{1,2} \cdot c_{2,4} \cdot c_{2,3 \mid 1} \cdot c_{1,4 \mid 2} \cdot c_{3,4 \mid 1,2} .
$$

The unwinding of this algorithm can yield other vine copulas if other parameters are used. For instance the following formula is another possible vine copula.

$$
c_{1,2,3,4}=c_{2,3} \cdot c_{3,4} \cdot c_{1,4} \cdot c_{2,4 \mid 3} \cdot c_{1,3 \mid 4} \cdot c_{1,2 \mid 3,4}
$$

And its associated tree:

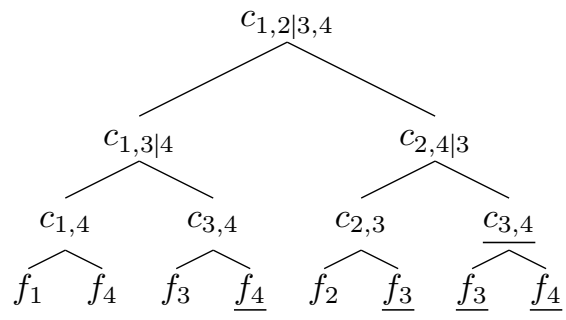




\section{Vine Analysis}

This section addresses the question of the estimation of a vine copula. For instance if we estimate the vine copula density $c_{1,2,3,4}$ constructed above with a 4-dimensional vector $\left(X_{1}, X_{2}, X_{3}, X_{4}\right)$, we need to estimate the bivariate copulas: $c_{1,3}, c_{1,2}, c_{2,4}$ and the bivariate conditional copula densities: $c_{2,3 \mid 1}, c_{1,4 \mid 2}$ and $c_{3,4 \mid 1,2}$. To estimate the formers we use standard methods as described in Patton (2009). We now turn to the problem of estimating the latters. Recall that for instance:

$$
c_{3,4 \mid 1,2}=c_{3,4 \mid 1,2}\left(F_{3 \mid 1,2}\left(X_{3} \mid X_{1}, X_{2}\right), F_{4 \mid 1,2}\left(X_{4} \mid X_{1}, X_{2}\right)\right) .
$$

Thus, to estimate $c_{3,4 \mid 1,2}$, we need to estimate $F_{3 \mid 1,2}$ and $F_{4 \mid 1,2}$. These conditional distribution functions can be built as follows: for $i, k_{1}, \ldots, k_{p} \in \llbracket 1, n \rrbracket^{p+1}$ and $j \in \llbracket 1, p \rrbracket$ :

$$
F_{i \mid k_{1}, \ldots, k_{p}}=\frac{\partial C_{i, k_{j} \mid k_{1} \ldots, k_{j-1}, k_{j+1}, \ldots, k_{p}}}{\partial F_{k_{j} \mid k_{1} \ldots, k_{j-1}, k_{j+1}, \ldots, k_{p}}},
$$

(Joe, 1997). We use the previous tree algorithm to choose the copula $C$ in formula (6). In our example to compute $F_{3 \mid 1,2}$ we will use $C_{2,3 \mid 1}$ and for $F_{4 \mid 1,2}$ we will use $C_{1,4 \mid 2}$.

\subsection{The Estimator}

Here we describe the statistical procedure to estimate vine copulas and provide asymptotic results. For $n \in \mathbb{N}, n>2$, we consider a $n$-variate vector $X=\left(X_{i}\right)_{i=1, \cdots n}$ and for all $i=1, \ldots, n$ we assume that we have an independent identically distributed (i.i.d) $T$-sample ${ }^{3}$. Our purpose is to estimate the parameter $\theta \in \Theta$ of the vine copula density $c_{1, \ldots, n}(. ; \theta)$. As soon as the random variables are i.i.d, we use the canonical maximum of likelihood method (White, 1994). We denote $\mathcal{L}$ the likelihood:

$$
\mathcal{L}(\theta)=\mathbb{E}_{T}\left[c_{1 \ldots, n}\left(F_{1}\left(X_{1}\right), \ldots, F_{n}\left(X_{n}\right) ; \theta\right)\right] .
$$

$\mathbb{E}_{T}$ denotes the sample expectation operator $\left(\mathbb{E}_{T}=T^{-1} \sum_{t=1}^{T}\right)$. We denote $\theta_{0}$ the pseudo true value of the parameter and $\hat{\theta}_{T}$ the maximum-likelihood estimator:

$$
\hat{\theta}_{T}=\underset{\theta \in \Theta}{\operatorname{argmax}} \mathcal{L}(\theta) .
$$

We introduce some technical assumptions:

- $A_{1}: \theta_{0}$ is interior to $\Theta$ and $\Theta$ is bounded.

- $A_{2}$ : All bivariate copula densities are bounded, $\mathcal{C}^{1}$ in $X$ and a.s $\mathcal{C}^{2}$ in their parameter ${ }^{4}$. In a neighborhood of $\theta_{0}$, all bivariate copula densities are $\mathcal{C}^{2}$ in their parameter.

\footnotetext{
${ }^{3}$ In practical applications, univariate models would be fit to the marginal process to filter the data into an i.i.d sample.

${ }^{4} \mathrm{~A}$ map $f$ is $\mathcal{C}^{k}$ if it is $k$ times differentiable and its $k$-th derivative is continuous.
} 
Theorem 1 Under assumptions $A_{1}$ and $A_{2}$ there exist a bounded matrix $V$ such that:

$$
\sqrt{T} V\left(\hat{\theta}_{T}-\theta_{0}\right) \stackrel{D}{\longrightarrow} N\left(0, I_{k}\right) .
$$

" $\stackrel{D}{\longrightarrow}$ " means convergence in distribution and $V=C_{0}^{1 / 2} \cdot H_{0}$ where $H_{0}=\left(H_{0 i, j}\right)_{i, j}$ with $H_{0 i, j}=\left.\frac{\partial^{2} \mathcal{L}}{\partial \theta_{i} \partial \theta_{j}}\right|_{\theta_{0}}$ and $C_{0}=\left(C_{0 i}\right)_{i}$ with $\left(C_{0}\right)_{i}=\left.\left(\frac{\partial \mathcal{L}}{\partial \theta_{i}}\right)^{2}\right|_{\theta_{0}}$.

Proof The proof can be found in the Annex.

This theorem provides the asymptotic normality of $\hat{\theta}_{T}$ with a $\sqrt{T}$ convergence rate. This result is central to any econometric applications. We denote now $\widehat{\operatorname{Cov}}\left(\hat{\theta}_{t}\right)$ the estimator of the covariance matrix of $\hat{\theta}_{T}$, and we have the following result:

Corollary 1 Under assumptions $A_{1}$ and $A_{2}$ :

$$
\begin{gathered}
\widehat{\operatorname{Cov}}\left(\hat{\theta}_{T}\right)=H^{-1} \cdot C \cdot H^{-1^{\prime}} . \\
H=\left(H_{i, j}\right)_{i, j} \text { with } H_{i, j}=\left.\frac{\partial^{2} \mathcal{L}}{\partial \theta_{i} \partial \theta_{j}}\right|_{\hat{\theta}_{T}} \text { and } C=\left(C_{i}\right)_{i} \text { with } C_{i}=\left.\left(\frac{\partial \mathcal{L}}{\partial \theta_{i}}\right)^{2}\right|_{\hat{\theta}_{T}} .
\end{gathered}
$$

Proof The proof can be found in the Annex.

Now we focus on the heterogeneity within the set of vine copulas in terms of asymptotic variance.

\subsection{Variance comparison}

In estimation theory when faced with the choice between two estimators we privilege the estimator with the smallest variance. Indeed such an estimator will require a smaller sample size $T$ to obtain significant results. Thus we compare the variance of two different vine copulas' estimators.

Theorem 2 Under assumptions $A_{1}, A_{2}$ and $A_{3}$. Let $\hat{\theta}_{T}$ and $\hat{\theta}_{T}^{\prime}$ be the estimator associated with two different vine copulas, then:

$$
\left\|\operatorname{Cov}\left(\hat{\theta}_{T}\right)-\operatorname{Cov}\left(\hat{\theta}_{T}^{\prime}\right)\right\|<\epsilon,
$$

where $\epsilon$ is a small real number. 
Proof The proof can be found in the Annex where we specify assumption $A_{3}$ and provide details on the choice of $\epsilon$.

The consequence of this theorem is that there is no evidence indicating that one should favor one type of vine copula over another. This result has important practical consequences that are discussed in Guégan and Maugis (2010).

The previous results confirm that we have a good estimators for vine copulas in terms of rate of convergence and that all vine copulas have comparable variance, thus their use is justified for applications. We now provide such an example.

\section{Application to the $C A C 40$ index}

Given the five main assets composing the $C A C 40$, the French leading index, using the methodology described above, we estimate their joint density in order to compute the $V a R^{5}$ of a portfolio composed of the five assets.

The dataset is taken from Datastream, daily quotes from Total, BNP - Paribas, Sanofi - Synthelabo, GDF-Suez and France Telecom from 25/4/08 to 21/11/08. This period is marked by the 2008 crisis, and our purpose is to test the resilience of our model to this shock and change of regimes. We estimate the parameters and select the vine copula based on the data ranging from 25/4/08 to $9 / 12 / 08$, while the $V a R$ is computed from the remaining dates. The portfolio we consider is as follows: Total (33\%), BNP - Paribas (20\%), Sanofi - Synthelabo (20\%), GDF-Suez (14\%) and France Telecom (13\%).

For each dataset a $\operatorname{GARCH}(p, q)$ process is selected using the $A I C$ criterion (Akaike, 1974) and estimated using pseudo likelihood. On the residuals we estimated the vine copula parameters using maximum likelihood. The parametric copula families used in this exercise are chosen among a panel of copulas which take into account most features commonly found in financial time series (Patton, 2009): they are the Clayton, Gumbel, Student and Gaussian copulas.

We select the best vine copula using the methodology described in Guégan and Maugis (2010) with the second test described in Chen et al. (2004). We recall that this test associates to each estimated density a $\chi^{2}$ sample. In this application, the retained vine copula is ${ }^{6}$ :

$$
c_{1,2,3,4,5}=c_{2,5} \cdot c_{4,5} \cdot c_{1,3} \cdot c_{1,5} \cdot c_{1,5 \mid 4} \cdot c_{1,2 \mid 5} \cdot
$$

\footnotetext{
${ }^{5}$ Given a random variable $X$, the $10 \% \operatorname{VaR}$ of $X$ is the value $\operatorname{VaR}(X)$ such that:

$$
P(X<\operatorname{VaR}(X))=0.1 \text {. }
$$

${ }^{6}$ The computation took one hour on a $1.5 G h z$ processor computer.
} 
Our final objective is to use this estimated vine copula density to compute the $10 \% \mathrm{VaR}$. We computed it from 9/12/08 to 21/11/08 using Monte-Carlo based integration and optimisation, see Figure 1. We compared it to a univariate $\operatorname{GARCH}(p, q)$ model-based estimate of the $V a R$ computed directly on the portfolio value time series in the same fashion as Samia et al. (2009). To discriminate between the two approaches, we use the Kupiec test (Kupiec, 1995). The Kupiec statistic is the number $Q$ of times the out-sample time-series is below the predicted $10 \% \mathrm{VaR}$. Under the null of the prediction being a true $10 \%$ $V a R$ the sampling distribution of the statistic follows a binomial distribution of parameter 0.1 .

In our example the vine copula- $V a R$ has a $p$-value of 0.96 for the $Q$ statistic, so it is accepted as a true $V a R$, while the $G A R C H$ - $V a R$ has a $p$-value of 0.00 and is rejected according to this test ${ }^{7}$. Nevertheless, the vine copula approach fails to predict the major drop during the crisis, but the prediction remains solid before and after the crisis. These results make the vine copula methodology we described an interesting approach for risk management in order to estimate the multivariate $(n>2)$ density of a portfolio and to compute its associated $V a R$.

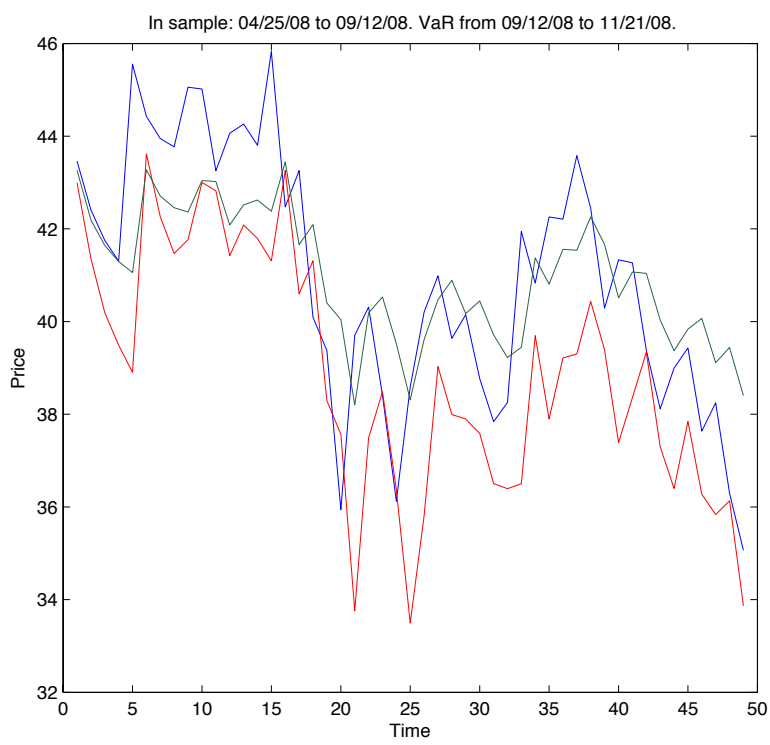

Figure 1: In Blue: The CAC40, In Red: Vine VaR Estimation, In Green: GARCH VaR Estimation.

\footnotetext{
${ }^{7} Q$ for the vine copula- $V a R$ is equal to 5 and for the $G A R C H-V a R$ is equal to 21.
} 


\section{Conclusion}

This paper focuses on the building of vines copulas using a tree-based algorithm. We provide the asymptotic normality of the vine copula parameter estimate under regular conditions. We show that, in the case of two competing vine copulas, no vine copula is better than another one in terms of variance criteria. This work provides solid statistical ground for the ideas developed in Aas et al. (2009) and Berg and Aas (2010) and justifies the methodologies used in Czado et al. (2009), Fischer et al. (2007), Chollete et al. (2008) and Guégan and Maugis (2010). Moreover, proving that no vine copula or sub-family of vine copula yields better estimator than others, justifies the use of all $N$ vine copulas which - as shown in Guégan and Maugis (2010) - enhances vine copulas capacity to represent more varied distributions. Finally, an application shows vine copulas usefulness to estimate $V a R$ and provides new and interesting risk management strategies for managers working with high dimensional portfolios.

Our results opens the possibility for varied uses of vines copulas. Most interestingly, the use of conditional copulas as described in Patton (2006), permits to relax the conditional independence hypothesis common throughout the vine copulas literature. This allows for very varied and interesting applications in all fields of economics and risk management.

\section{Acknowledgment}

This work has already been presented at the MIT Econometric Seminar (Cambridge, USA, 2009) and at the International Symposium in Computational Economics and Finance (Sousse, Tunisia, 2010). We thank the participants of the seminars and our reviewers for their helpful comments. All remaining errors are still ours. P.A. Maugis thank Arun Chandrasekhar, Victor Chernozhukov and Miriam Sofronia for their helpful comments.

\section{References}

Aas, K., Czado, C., Frigessi, A., Bakken, H., 2009. Pair-copula constructions of multiple dependence. Insurance: Mathematics and Economics 44, 182-198.

Akaike, H., 1974. A new look at the statistical model identification. IEEE Trans. Automatic Control AC-19, 716-723.

Artzner, P., Delboen, F., Eber, J., Heath, D., 1997. Thinking coherency. Risk $10,68-71$. 
Bedford, T., Cooke, R., 2001. Probability density decomposition for conditionally dependent random variables modeled by vines. Annals of Mathematics and Artificial Intelligence 32, 245-268.

Bedford, T., Cooke, R., 2002. Vines: A New Graphical Model for Dependent Random Variables. The Annals of Statistics 30 (4), 1031-1068.

Berg, D., Aas, K., 2010. Models for construction of multivariate dependence. Forthcoming in The Europen Journal of Finance.

Chen, X., Fan, Y., Patton, A., 2004. Simple Tests for Models of Dependence Between Multiple Financial Time Series, with Applications to U.S. Equity Returns and Exchange Rates. London Economics Financial Markets Group Working Paper 483, london, UK.

Chollete, L., Andržas, H., Valdesogo, A., 2008. Modeling international financial returns with a multivariate regime switching copula. Journal of Financial Econometrics 7, 437-480.

Cooke, R. (Ed.), 1997. Markov and entropy properties of tree- and vinedependent variables. American Statistical Association Section on Bayesian Statistical Science, Alexandria, VA.

Czado, C., Gartner, F., Min, A., 2009. Analysis of Australian electricity loads using joint Bayesian inference of D-Vines with autoregressive margins. Zentrum Mathematik Technische Universitat Munchen: Working Paper Munchen, Germany.

Fischer, M., Köck, C., Schlü̈er, S., Weigert, F., 2007. Multivariate copula models at work: Outperforming the desert island copula? Tech. Rep. 79, Universität Erlangen-Nü̈nberg, Lehrstuhl für Statistik und Ö̈onometrie.

Guégan, D., Maugis, P. A., 2010. Prospects On Vines. Forthcoming in Insurance Markets and Companies: Analyses and Actuarial Computations.

Joe, H., 1997. Multivariate Models and Dependence Concepts. Chapman \& Hall, London, UK.

Kupiec, P., 1995. Techniques for verifying the accuracy of risk measurement models. Board of Governors of the Federal Reserve System (U.S.), Washington 24 (95).

Napoles, O. M. (Ed.), 2007. Number Of Vines. 1st Vine Copula Workshop, Delft, Netherland.

Patton, A., 05 2006. Modelling asymmetric exchange rate dependence. International Economic Review 47 (2), 527-556.

Patton, A., 2009. Handbook of Financial Time Series, Chapter V-3. Springer Verlag, Ch. Copula-Based Models for Financial Time Series, pp. 767-781. 
Samia, M., Dalenda, M., Saoussen, A., 2009. Accuracy and Conservatism of $V a R$ Models: A Wavelet Decomposed VaR Approach Versus Standard $A R M A-G A R C H$ Method. International Journal of Economics and Finance 1 (2), 174-184.

Sklar, A., 1959. Fonctions de répartition à $n$ dimensions et leurs marges. Publications de l'Institut de Statistique de L' Université de Paris 8, 229-231.

White, H., 1994. Estimation, Inference and Specification Analysis. Cambridge University Press: Cambridge.

\section{Annex}

\subsection{Proof of Theorem 1}

Before providing the proof of Theorem 1, we introduce some definitions and notations.

\subsubsection{Definitions and Notations}

Let $n \in \mathbb{N}, n>2$ and $X=\left(X_{i}\right)_{i=1, \cdots n}$. For all $i$ we consider an independent identically distributed $T$-sample.

- We note $\mathbb{E}$ the distribution expectation operator and $\mathbb{E}_{T}$ the sample expectation operator $\left(\mathbb{E}_{T}=T^{-1} \sum_{t=1}^{T}\right)$.

- We use the del operator $\nabla$ : for a function $f\left(x_{1}, \ldots, x_{n}\right), \nabla f$ is equal to $\left(\partial_{x_{1}} f, \ldots, \partial_{x_{n}} f\right)$.

- For $i, j, k_{1}, \ldots, k_{p} \in \llbracket 1, n \rrbracket^{p+2}$, we call $s=\left\{i, j \mid k_{1}, \ldots, k_{p}\right\}$ the index of the following copula density:

$$
c_{s}=c_{i, j \mid k_{1}, \ldots, k_{p}}\left(F\left(X_{i} \mid\left(X_{l}\right)_{l=k_{1}, \ldots, k_{p}}\right), F\left(X_{j} \mid\left(X_{l}\right)_{l=k_{1}, \ldots, k_{p}}\right)\right) .
$$

For instance $\{2,3 \mid 1\}$ is the index of $c_{2,3 \mid 1}\left(F\left(x_{2} \mid x_{1}\right), F\left(x_{3} \mid x_{1}\right)\right)$. 
- To each vine copula we associate a set $M$. Let $M$ be the set of the indexes of the bivariate copulas and marginal densities used to estimated the associated vine copula. It is also the set of the labels of the nods of the tree associated with a vine copula. We call $\mathcal{M}_{n}$ the set of all possible models in dimension $n$.

For instance for $n=3$ :

$$
f_{1,2,3}=f_{1} \cdot f_{2} \cdot f_{3} \cdot c_{1,2} \cdot c_{1,3} \cdot c_{2,3 \mid 1}
$$

Then the model $M$ associated with this vine copula is:

$$
M=\{\{1\},\{2\},\{3\},\{1,2\},\{1,3\},\{2,3 \mid 1\}\} .
$$

- We introduce respectively the following parametric families: for each univariate processes, bivariate copulas and conditional copula parameter,

$$
\left\{\gamma(. ; \theta) \mid \theta \in \Theta^{\prime}\right\} ;\left\{\varphi(., . ; \theta) \mid \theta \in \Theta^{\prime \prime}\right\} ;\left\{\xi(. ; \theta) \mid \theta \in \Theta^{\prime \prime \prime}\right\} .
$$

We now construct the likelihood functions used to estimate the parameters. For $M \in \mathcal{M}_{n}$ we define the likelihood functions $\left\{\psi_{s}^{M}\right\}_{s \in M}$ as:

$$
\begin{aligned}
& \forall i, j, k, \in \llbracket 1, n \rrbracket^{3} i \neq j \neq k, \forall \theta=\left(\theta_{i}, \theta_{j}, \theta_{i, k}, \theta_{j, k}, \theta_{i, j \mid k}\right) \in \Theta^{\prime 2} \times \Theta^{\prime \prime 2} \times \Theta^{\prime \prime \prime}: \\
& \psi_{i}^{M}(\theta)=\mathbb{E}_{T}\left[\log \gamma^{M}\left(X_{i} ; \theta_{i}\right)\right] \\
& \begin{array}{l}
\psi_{i, j}^{M}(\theta)=\mathbb{E}_{T}\left[\log \varphi^{M}\left(F_{i}^{M}\left(X_{i} ; \theta_{i}\right), F_{j}^{M}\left(X_{j} ; \theta_{j}\right) ; \theta_{i, j}\right)\right] \\
\psi_{i, j \mid k_{T}}^{M}(\theta)=
\end{array} \\
& \mathbb{E}_{T}\left[\log \varphi^{M}\left(F_{i \mid k}^{M}\left(X_{i} \mid X_{k} ; \theta_{i}, \theta_{k}, \theta_{i, k}\right), F_{j \mid k}^{M}\left(X_{j} \mid X_{k} ; \theta_{j}, \theta_{k}, \theta_{j, k}\right) ; \xi\left(X_{k} ; \theta_{i, j \mid k}\right)\right)\right] \\
& \text {.. } \quad=\ldots
\end{aligned}
$$

We use $M$ as superscript to denote that the estimation is done according to the model $M$. The conditional distribution functions are computed using formula (6). Using the previous notations the log-likelihood is equal to:

$$
\mathcal{L}^{M}(\theta)=\sum_{s \in M} \psi_{s}^{M}(\theta) .
$$

Then:

$$
\begin{aligned}
\hat{\theta}_{T}^{M} & =\underset{\theta \in \Theta}{\operatorname{argmax}} \mathcal{L}^{M}(\theta) \\
\theta_{0}^{M} & =\left[\theta_{M(i)}^{0}\right]_{i \in \llbracket 1,|M| \rrbracket}
\end{aligned}
$$




\subsubsection{Assumptions}

To prove the convergence we make some assumptions that are verified for a vast majority of parametric copula families:

- $\theta_{0}$ is interior to $\Theta$, the set of possible parameters.

- $\forall s \in M \psi_{s}^{M}, \nabla_{\theta, \theta} \psi_{s}^{M}$ and $\nabla_{\theta, \theta}^{2} \psi_{s}^{M}$ are bounded and a.s uniformly continuous in $\theta$ and uniformly continuous in a neighborhood of $\theta_{0}$.

- All $\varphi$ and $\xi$ used for estimation are a.s $\mathcal{C}^{1}$ in $(X)_{i \leq n}$ and a.s $\mathcal{C}^{2}$ in $\theta$ and are $\mathcal{C}^{1}$ in $(X)_{i \leq n}$ and $\mathcal{C}^{2}$ in $\theta$ in a neighborhood of $\theta_{0}$.

These assumptions are weaker than assumptions $A_{1}$ and $A_{2}$ and are implied by $A_{1}$ and $A_{2}$.

We introduce the exponent 0 to specify the function are evaluated at $\theta_{0}^{M}$, and denote:

$\varphi_{i, j \mid k}^{0}=\varphi\left(F_{i \mid k}^{M}\left(X_{i} \mid X_{k} ;\left\{\theta_{0}^{M}\right\}_{i, k,\{i, k\}}\right), F_{j \mid k}^{M}\left(X_{j} \mid X_{k} ;\left\{\theta_{0}^{M}\right\}_{j, k,\{j, k\}}\right) ; \xi\left(X_{k} ; \theta_{0}^{M}{ }_{i, j \mid k}\right)\right)$.

Also:

$$
\varphi_{i}{ }^{M, 0}=\varphi^{M}\left(X_{i} ; \theta_{i}^{0}\right),{ }^{M} \varphi_{i, j_{t}}^{0}=\varphi^{M}\left(F^{M}\left(X_{i}\right), F^{M}\left(X_{j}\right) ; \theta_{i}^{0}, \theta_{j}^{0}, \theta_{i, j}^{0}\right),
$$

when we work at $\theta_{0}^{M}$.

\subsubsection{Operators}

To compute the derivates of $\mathcal{L}^{M}$, we use the following property:

$$
\forall M \in \mathcal{M}_{n}, \forall i \in \llbracket 1,|M| \rrbracket, \forall s \in M \backslash m(M(i)), \quad \nabla_{\theta_{M(i)}} \psi_{s}^{M} \equiv 0 .
$$

Indeed if $s \in M \backslash m(M(i))$ then $\psi_{s T}^{M}$ is not dependent in $\theta_{M(i)}$ so that the gradient is null.

We introduce ${ }^{M} \mathrm{Grad}_{T}$ as the gradient of $\mathcal{L}^{M}$ :

$$
\begin{aligned}
{ }^{M} \operatorname{Grad}_{T} & =\nabla \mathcal{L}^{M}=\left(\nabla_{\theta_{M(i)}} \mathcal{L}^{M}\right)_{i \in \llbracket 1,|M| \rrbracket} \\
& =\left(\nabla_{\theta_{M(i)}} \sum_{s \in m(M(i))} \psi_{s}^{M} T\right)_{i \in \llbracket 1,|M| \rrbracket}
\end{aligned}
$$


We define ${ }^{M} \mathrm{Hess}_{T}$, the Hessian matrix of $\mathcal{L}^{M}$ :

$$
\begin{aligned}
{ }^{M} \text { Hess }_{T} & =\nabla^{2} \mathcal{L}^{M}=\left(\nabla_{\theta_{M(i)}, \theta_{M(j)}} \mathcal{L}^{M}\right)_{i, j \in \llbracket 1,|M| \rrbracket^{2}} \\
& =\left(\nabla_{\theta_{M(i)}, \theta_{M(j)}} \sum_{s \in m(M(i)) \cap m(M(j))} \psi_{s T}^{M}\right)_{i, j \in \llbracket 1,|M| \rrbracket^{2}}
\end{aligned}
$$

We denote ${ }^{M} C_{T}^{0}$ the covariance matrix of $\sqrt{T} \cdot{ }^{M} \operatorname{Grad}_{T}\left(\theta_{0}\right)$ :

$$
\begin{aligned}
{ }^{M} C_{T}^{0} & =\left(\mathbb{E}_{T}\left(\left(\sum_{s \in M} \nabla_{\theta_{M(i)}} \log \varphi_{s}^{0^{M}}\right)\left(\sum_{s \in M} \nabla_{\theta_{M(j)}} \log \varphi_{s}^{0^{M}}\right)\right)\right)_{i, j \in[1,|M|]^{2}} \\
& =\left(\mathbb{E}_{T}\left(\sum_{s \in m(M(i)) \cap m(M(j))} \nabla_{\theta_{M(i)}} \log \varphi_{s}^{0^{M}} \nabla_{\theta_{M(j)}} \log \varphi_{s}^{0^{M}}\right)\right)_{i, j \in[1,|M|]^{2}}
\end{aligned}
$$

Finally we define $H_{0}^{M}$ and $C_{0}^{M}$ as follows:

$$
H_{0}^{M}=\mathbb{E}\left[{ }^{M} \operatorname{Hess}_{T}^{0}\right] ; C_{0}^{M}=\mathbb{E}\left[{ }^{M} C_{T}^{0}\right]
$$

\subsubsection{Rate of Convergence}

We now prove that the rate of convergence for the maximum likelihood estimate $\hat{\theta}_{T}^{M}$ is $\sqrt{T}$ for all models $M$ :

$$
0={ }^{M} \operatorname{Grad} d_{T}^{0}\left(\hat{\theta}_{T}^{M}\right)={ }^{M} \operatorname{Grad} d_{T}^{0}\left(\theta_{0}^{M}\right)+{ }^{M} \operatorname{Hess}_{T}^{0}\left(\bar{\theta}_{T}\right)\left(\hat{\theta}_{T}^{M}-\theta_{0}^{M}\right)
$$

Where $\bar{\theta}_{T}$ belongs to $\left[\hat{\theta}_{T}^{M}, \theta_{0}^{M}\right]$

$$
\begin{aligned}
{ }^{M} \operatorname{Hess}_{T}^{0}\left(\bar{\theta}_{T}\right)\left(\hat{\theta}_{T}^{M}-\theta_{0}^{M}\right) & =-{ }^{M} \operatorname{Grad}_{T}^{0}\left(\theta_{0}^{M}\right) \\
\sqrt{T} C_{0}^{M^{-1 / 2}} \cdot{ }^{M} \operatorname{Hess}_{T}^{0}\left(\bar{\theta}_{T}\right)\left(\hat{\theta}_{T}^{M}-\theta_{0}^{M}\right) & =-C_{0}^{M^{-1 / 2}} \sqrt{T^{M}} \operatorname{Grad}_{T}^{0}\left(\theta_{0}^{M}\right) \\
\sqrt{T} C_{0}^{M^{-1 / 2}} \cdot H_{0}^{M}\left(\hat{\theta}_{T}^{M}-\theta_{0}^{M}\right) & =-C_{0}^{M^{-1 / 2}} \sqrt{T}{ }^{M} \operatorname{Grad}_{T}^{0}\left(\theta_{0}^{M}\right)+o_{p}(1) \\
\sqrt{T} C_{0}^{M^{-1 / 2}} \cdot H_{0}^{M}\left(\hat{\theta}_{T}^{M}-\theta_{0}^{M}\right) & \stackrel{D}{\rightarrow}\left(0, I_{k}\right)
\end{aligned}
$$

Using the central limit theorem and the uniform bounds on $\psi$ 's derivatives we obtain the convergence in distribution (we can use these bounds because $\theta_{0}$ is interior to $\Theta$ ). The proof of Theorem 1 is complete.

\subsection{Proof of Theorem 2}

Before providing the proof of Theorem 2, we introduce some definitions and notations. 


\subsubsection{Defininition}

- For each vine decomposition we define a function $m$ that associates to each element $s$ in $M$ the set of indexes of bivariate copulas and marginal densities necessary to estimates $c_{s}$

$$
m: M \rightarrow \mathcal{P}(M)
$$

Where $\mathcal{P}(M)$ is the set of subsets of $M$.

- For all $M$ in $\mathcal{M}_{n}$ we order their elements according to the underlying tree structure. $M(1)$ is the index of the copula which is at the root of the tree, $M(2)$ is the index of the copula of left leaf from the root of the tree, $M(3)$ is the label of the copula of the right leaf of the tree, and so on.

Example Consider the following trivariate vine copula:

$$
f_{1,2,3}=\overbrace{f_{f_{1} f_{f_{2}}} \overbrace{f_{f_{1}}^{c_{1,3}} f_{3}}^{c_{2,3 \mid 1}} \cdot f_{2} \cdot f_{3} \cdot c_{1,2} \cdot c_{1,3} \cdot c_{2,3 \mid 1}}^{c_{1,2}}
$$

Then the map $m$ and the indexes in $M($.$) defined above are:$

$$
\begin{aligned}
& m(\{1\})=\{1\} ; m(\{2\})=\{2\} ; m(\{3\})=\{3\} \\
& m(\{1,2\})=\{\{1\},\{2\},\{1,2\}\} ; m(\{1,3\})=\{\{1\},\{3\},\{1,3\}\} \\
& m(\{2,3 \mid 1\})=\{\{1\},\{2\},\{3\},\{1,2\},\{1,3\},\{2,3 \mid 1\}\} \\
& M(1)=\{2,3 \mid 1\} ; M(2)=\{1,2\} ; M(3)=\{1,3\} \\
& M(4)=\{1\} ; M(5)=\{2\} ; M(6)=\{3\}
\end{aligned}
$$

\subsubsection{Proof}

The key point in this analysis is the following: as soon as the ordering of the model $M$ is done according to the tree structure, and this structure is the same for all models, then:

$$
\begin{aligned}
& \forall M, M^{\prime} \in \mathcal{M}_{n}^{2}, \forall i, j \in \llbracket 1,|M| \rrbracket^{2} \\
& M^{-1}(m(M(i)) \cap m(M(j)))=M^{\prime-1}\left(m^{\prime}\left(M^{\prime}(i)\right) \cap m^{\prime}\left(M^{\prime}(j)\right)\right),
\end{aligned}
$$


where $M^{-1}$ denotes the function that maps a copula to its index number in $M$. This equality links the two copulas numbered $i$ and $j$ through the tree structure. We now define $K_{i, j}$ and $k_{i, j}$ as follows:

$$
\begin{array}{ll}
\forall M \in \mathcal{M}_{n}, \forall i, j \in \llbracket 1,|M| \rrbracket^{2} & K_{i, j}=m(M(i)) \cap m(M(j)), \\
\forall M \in \mathcal{M}_{n}, \forall i, j \in \llbracket 1,|M| \rrbracket^{2} & k_{i, j}=|m(M(i)) \cap m(M(j))| .
\end{array}
$$

This definition allows us to control the covariance matrix of two different models by controlling the difference of each term of the sums over $K_{i, j}$ for all $i$ and $j$. We now introduce a distance that permits to compare two models $M$ and $M^{\prime}$ :

\section{Definition}

- $\simeq_{\epsilon}$ is defined by:

$$
\forall A, B \in E^{2}(\mathbb{R},\|\cdot\|) \quad A \simeq_{\epsilon} B \Leftrightarrow\|A-B\| \leq \epsilon \Leftrightarrow \exists \nu \in E, A=B+\nu,\|\nu\| \leq \epsilon .
$$

- Let $\epsilon$ be the the smallest real number such that:

$$
\begin{aligned}
& \forall M, M^{\prime} \in \mathcal{M}_{n}^{2}, \forall i, j, r \in \llbracket 1,|M| \rrbracket^{3} \\
& \left\{\begin{array}{c}
\mathbb{E}_{T}\left(\nabla_{\theta_{M(i)}} \log \varphi_{M(r)}^{0}{ }^{M} \nabla_{\theta_{M(j)}} \log \varphi_{M(r)}^{0}{ }^{M}\right) \simeq_{\epsilon} \\
\mathbb{E}_{T}\left(\nabla_{\theta_{M^{\prime}(i)}} \log \varphi_{M^{\prime}(r)}^{0}{ }^{M^{\prime}} \nabla_{\theta_{M^{\prime}(j)}} \log \varphi_{M^{\prime}(r)}^{0}{ }^{M^{\prime}}\right), \\
\nabla_{\theta_{M(i)}, \theta_{M(j)}} \psi_{M(r)}^{M, 0} \simeq_{\epsilon} \nabla_{\theta_{M(i)}, \theta_{M(j)}} \psi_{M(r)}^{M^{\prime}, 0} .
\end{array}\right.
\end{aligned}
$$

In practice $\epsilon$ is small as both pairs of continuous functions $\varphi_{M(r)}^{M}, \varphi_{M^{\prime}(r)}^{M^{\prime}}$ and $\psi_{M(r)}^{M}, \psi_{M^{\prime}(r)}^{M^{\prime}}$ are equal if the same parametric pair copula is used throughout the estimation, which is the case in most applications (Aas et al. (2009), Berg and Aas (2010) and Czado et al. (2009)).

\section{Assumption $A_{3}$}

$$
\exists M \in \mathcal{M}_{n}, \text { s.t } \epsilon<\left\|^{M} H e s s_{T}^{0}\right\| /\left\|\left(k_{i, j}\right)_{i, j}\right\|
$$

Using the previous assumptions and the definition made above we can write ${ }^{M} \mathrm{Hess}_{T}^{0}$ as follows:

$$
\begin{aligned}
{ }^{M} \mathrm{Hess}_{T}^{0} & =\left(\nabla_{\theta_{M(i)}, \theta_{M(j)}} \sum_{s \in m(M(i)) \cap m(M(j))} \psi_{s}^{M^{0}}\right)_{i, j \in \llbracket 1,|M| \rrbracket^{2}} \\
& =\left(\nabla_{\theta_{M(i)}, \theta_{M(j)}} \sum_{s \in K_{i, j}} \psi_{s}^{M^{0}}\right)_{i, j \in \llbracket 1,|M| \rrbracket^{2}} \\
& =\left(\nabla_{\theta_{M^{\prime}(i)}, \theta_{M^{\prime}(j)}} \sum_{s \in K_{i, j}} M^{\prime} \psi_{s T}^{0}\right)_{i, j \in \llbracket 1,|M| \rrbracket^{2}}+\left(\nu_{i, j} k_{i, j}\right)_{i, j \in \llbracket 1,|M| \rrbracket^{2}} \\
& \simeq_{\epsilon \cdot\left\|\left(k_{i, j}\right)_{i, j}\right\|}{ }^{M^{\prime}} \operatorname{Hess}_{T}^{0} .
\end{aligned}
$$


And ${ }^{M} C_{T}^{0}$ can also be rewritten in the following way:

$$
\begin{aligned}
{ }^{M} C_{T}^{0} & =\left(\mathbb{E}_{T}\left(\sum_{s \in m(M(i)) \cap m(M(j))} \nabla_{\theta_{M(i)}} \log \varphi_{s}^{0^{M}} \nabla_{\theta_{M(j)}} \log \varphi_{s}^{0^{M}}\right)\right)_{i, j \in \llbracket 1,|M|]^{2}} \\
& =\left(\mathbb{E}_{T}\left(\sum_{s \in K_{i, j}} \nabla_{\theta_{M(i)}} \log \varphi_{s}^{0^{M}} \nabla_{\theta_{M(j)}} \log \varphi_{s}^{0^{M}}\right)\right)_{i, j \in \llbracket 1,|M| \rrbracket^{2}} \\
& \simeq_{\epsilon \cdot\left\|\left(k_{i, j}\right)_{i, j}\right\|}\left(\mathbb{E}_{T}\left(\sum_{s \in K_{i, j}} \nabla_{\theta_{M^{\prime}(i)}} \log \varphi_{s M^{\prime}}^{0} \nabla_{\theta_{M^{\prime}(j)}} \log \varphi_{s M^{\prime}}^{0}\right)\right)_{i, j \in \llbracket 1,|M| \rrbracket^{2}} \\
& \simeq_{\epsilon \cdot\left\|\left(k_{i, j}\right)_{i, j}\right\|}{ }^{M^{\prime}} C_{T}^{0}
\end{aligned}
$$

In order to establish formula (7) we will use shorthand notations ${ }^{8}$.

$$
\begin{aligned}
K=\left(k_{i, j}\right)_{i, j} ; & H={ }^{M} \operatorname{Hess}_{t}^{0} ; C={ }^{M} C_{T}^{0} ; V=H^{-1} C H^{\prime}-1 ; V_{0}=H^{-1} K H^{\prime-1} ; I=I d_{|M|} \\
\widehat{\operatorname{Cov}}_{T}^{M^{\prime}} & =\left(H+\nu_{1} K\right)^{-1}\left(G+\nu_{2} K\right)\left(H+\nu_{1} K\right)^{\prime}-1 \\
& =\left(I+\nu_{1} H^{-1} K\right)^{-1} H^{-1}\left(G+\nu_{2} K\right) H^{-1}\left(I+\nu_{1} K H^{-1}\right)^{\prime}-1 \\
& =\left(I+\sum_{i=1}^{\infty}(-1)^{i}\left(\nu_{1} H^{-1} K\right)^{i}\right)\left(V+\nu_{2} V_{0}\right)\left(I+\sum_{i=1}^{\infty}(-1)^{i}\left(\nu_{1} H^{-1} K\right)^{i}\right)^{\prime} \\
& =\left(I+\Sigma_{\nu_{1}}\right)\left(V+\nu_{2} V_{0}\right)\left(I+\Sigma_{\nu_{1}}\right)^{\prime} \\
& =V+\nu_{2} V_{0}+2 \Sigma_{\nu_{1}}\left(V+\nu_{2} V_{0}\right)+\Sigma_{\nu_{1}}{ }^{2}\left(V+\nu_{2} V_{0}\right) \\
& <\alpha \cdot \epsilon
\end{aligned}
$$

The proof of theorem 2 is complete.

\footnotetext{
${ }^{8}$ In Theorem 2 we noted $\epsilon$ the value $\alpha \cdot \epsilon$
} 